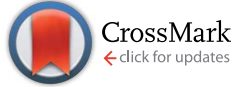

Cite this: RSC Adv., 2016, 6, 55211

Received 30th March 2016 Accepted 28th May 2016

DOI: $10.1039 / c 6 r a 08234 c$

www.rsc.org/advances

\section{Antimicrobial hydrogels based on autoclaved poly(vinyl alcohol) and poly(methyl vinyl ether-alt- maleic anhydride) mixtures for wound care applications $\uparrow$}

\begin{abstract}
Enrica Caló, ${ }^{a}$ Joao M. S. de Barros, ${ }^{a}$ Mar Fernández-Gutiérrez, ${ }^{\text {bc }}$ Julio San Román, Lucy Ballamy ${ }^{d}$ and Vitaliy V. Khutoryanskiy ${ }^{\star a}$

Novel antimicrobial hydrogels with good mechanical and physical properties were synthesized by autoclaving aqueous mixtures of poly(vinyl alcohol) and poly(methyl vinyl ether-alt-maleic anhydride). The structure of these materials was studied by infrared spectroscopy, scanning electron microscopy and solid state nuclear magnetic resonance. The swelling behavior, mechanical properties and adhesion of the hydrogels to porcine skin were evaluated. It was established that these hydrogels exhibited antimicrobial properties and inhibited bacteria growth against Staphylococcus aureus. The biocompatibility of the hydrogels was confirmed using an MTT assay (indirect cytotoxicity) and by monitoring cell proliferation in contact with the gels (direct cytotoxicity).
\end{abstract}

\section{Introduction}

Hydrogels are three-dimensional networks produced by chemical (chemical hydrogels) or physical (physical hydrogels) crosslinking of water-soluble polymers, which can be achieved using different synthetic approaches. ${ }^{1}$ The starting materials for making hydrogels can be represented by natural or synthetic monomers or polymers that can form hydrophilic networks alone, or with the addition of cross-linking agents or/and initiators. ${ }^{2}$ Hydrogels are widely used as biomaterials for drug delivery, tissue engineering and wound dressings, due to their unique mechanical and physical properties. ${ }^{3-5}$

Hydrogels have drawn interest from the wound care industry because of their resemblance to living tissue as well as their ability to absorb and retain exudate which improves the balance of hydration of the wound bed., ${ }^{6,7}$ A major advantage of hydrogel products is their transparency which allows the user to visually monitor the wound without removing the dressing. Unlike traditional wound care products, such as common plain gauze, hydrogel dressings do not require daily changes. ${ }^{8,9}$ Their high

\footnotetext{
${ }^{a}$ Reading School of Pharmacy, University of Reading, Reading, Berkshire, RG6 6AD, UK. E-mail:v.khutoryanskiy@reading.ac.uk

${ }^{b}$ Institute of Polymer Science and Technology, CSIC, C/Juan de la Cierva 3, Madrid 28006, Spain

${ }^{c}$ CIBER-BBN, C/ Monforte de Lemos 3-5, Madrid 28029, Spain

${ }^{d}$ ConvaTec UK Ltd, First Avenue-Deeside Industrial Park, Flintshire CH5 2NU, Wales, $U K$

$\dagger$ Electronic supplementary information (ESI) available: Exemplary stress-strain and detachment curves from porcine skin curve; additional swelling kinetics results. See DOI: 10.1039/c6ra08234c
}

water content facilitates vapour and oxygen diffusion to the wound which may accelerate the healing process. ${ }^{9}$ Hydrogels are also well known to promote autolytic debridement, which is a process in which the body breaks down and removes dead tissue, carried out by specific enzymes called matrix metalloproteinases which migrate to the wound site after injury. ${ }^{\mathbf{1 0}}$

Hydrogel-based dressings can be designed as amorphous gels or as flat sheets ready to be applied. In both cases they conform and slightly adhere to the surface of the wound, therefore avoiding the formation of the so-called 'dead space' where bacteria can proliferate causing infections. ${ }^{11}$

Hydrogels are typically synthesized via three-dimensional polymerization of hydrophilic monomers. This method requires extensive purification of the hydrogel product from any unreacted monomers, which are often toxic. Purification is achieved by extraction using excess water and may take up to two weeks. An alternative method for the preparation of hydrogels is the cross-linking of water-soluble polymers. This is achieved by using ionizing radiation such as gamma rays or accelerated electrons. ${ }^{12}$ The advantage of this method is that the hydrogel formation and its sterilization are achieved in a single step. However, the application of radiation requires the use of expensive facilities.

Recently we pioneered the synthesis of hydrogels using microwave-mediated thermal cross-linking in aqueous mixtures of functional water-soluble polymers. This method offers the advantage of hydrogel formation with simultaneous sterilization without the need for their purification because no toxic monomers or cross-linkers are used. ${ }^{13}$ In microwave processing, energy is supplied by an electromagnetic field and results in 
rapid heating of aqueous solutions. The use of this method commercially is often limited because of the need for access to industrial microwave facilities. Autoclaving is a viable alternative to microwave processing and is a more common method of heating aqueous solutions at industrial scale. Autoclaving is widely used for sterilisation of medical devices.

The present work is the first report on the synthesis of 'readyto-use' hydrogels by autoclaving aqueous mixtures of poly(vinyl alcohol) (PVA) and poly(methyl vinyl ether-alt-maleic anhydride) (Gantrez® AN or Gant). The hydrogels developed in this work have a unique combination of properties such as excellent swelling ability, good mechanical strength, adhesiveness to skin, as well as intrinsic antimicrobial activity.

\section{Experimental section}

\subsection{Materials}

PVA of 98-99\% hydrolysis and of medium ( $\left.M_{\mathrm{w}} 57-66 \mathrm{kDa}\right)$ and high $\left(M_{\mathrm{w}}\right.$ 88-97 kDa) molecular weights were purchased from Alfa Aesar (UK). The hydrogel compositions will be then referred to as PVAm-Gant and PVAh-Gant (with their molar ratios) in figures and tables. Actual degree of PVA deacetylation was verified by ${ }^{1} \mathrm{H}$ NMR and found to be $98.7 \%$, and the actual $M_{\mathrm{w}}$ of the PVA used were found to be $51.3 \mathrm{kDa}$ (for medium $M_{\mathrm{w}}$ PVA) and $66.3 \mathrm{kDa}$ (for high $M_{\mathrm{w}}$ PVA) by gel permeation chromatography. Nutrient agar used in the antimicrobial test was purchased from Oxoid Ltd UK. Hoechst 33342 nucleic acid stain was purchased from Life Technologies (Molecular Probes ${ }^{\mathrm{TM}}$ ). Gantrez ${ }^{\circledR}$ AN $\left(M_{\mathrm{w}} \sim 216 \mathrm{kDa}\right)$ and all the other chemicals used were purchased from Sigma-Aldrich (UK). Aquacel ${ }^{\circledR} \mathrm{Ag}$ dressings (used as a control dressing during antimicrobial properties and biocompatibility tests) were kindly provided by ConvaTec Ltd.

\subsection{Synthesis of autoclaved hydrogels}

The aqueous mixtures of polymers were prepared using different PVA-Gantrez ${ }^{\circledR}$ AN molar ratios: $1: 1,1: 2,2: 1$ (base moles of PVA per base moles of Gantrez ${ }^{\circledR}$ AN). For hydrogel synthesis $17.4 \% \mathrm{w} / \mathrm{v}$ aqueous solution of Gantrez ${ }^{\circledR}$ AN was added to $4.4 \% \mathrm{w} / \mathrm{v}$ solutions of PVA (with a final volume of 15 $\mathrm{mL}$ ) and the mixtures were stirred for 205 minutes at room temperature. Then they were cast in $8 \mathrm{~cm}$ diameter glass Petri dishes which were autoclaved at $125{ }^{\circ} \mathrm{C}, 1.4$ bar for 90 minutes (CertoClav Multicontrol 12 L).

\subsection{Synthesis of freeze-thaw hydrogels}

Mixtures of $10 \% \mathrm{w} / \mathrm{v}$ aqueous solution of PVA and $17.4 \% \mathrm{w} / \mathrm{v}$ aqueous solution of Gantrez ${ }^{\circledR}$ AN with a $1: 2$ molar ratio of the two polymers, containing $0.25 \% \mathrm{w} / \mathrm{v}$ of $\mathrm{NaOH}$ (with a final volume of $18 \mathrm{~mL}$ ) were frozen at $-18{ }^{\circ} \mathrm{C}$ for 9 hours and then thawed at room temperature resulting in physically cross-linked hydrogels.

\subsection{Fourier transform infrared spectroscopy (FTIR)}

Fourier transform infrared spectra of PVA powder, Gantrez® AN powder and a freeze-dried gel sample were recorded using a Nicolet IS5 ID5 ATR infrared spectrometer and data analyzed using OMNIC software with 16 scans and a resolution of $4 \mathrm{~cm}^{-1}$.

\subsection{Solid state nuclear magnetic resonance (NMR)}

Hydrogel samples, and individual PVA and Gantrez ${ }^{\circledR}$ AN solutions were freeze-dried and used for recording solid state ${ }^{13} \mathrm{C}$ NMR spectra. ${ }^{13} \mathrm{C}$ solid state cross-polarization magic angle spinning (CPMAS) NMR spectra were recorded on Bruker Avance III spectrometer operating at Larmor frequency of 125.78 MHz (11.75 T). The standard bore $4 \mathrm{~mm}$ MAS probe was spun at $15 \mathrm{kHz}$ rate. The $\mathrm{CP}$ contact time was $1.4 \mathrm{~ms}$, and the $90^{\circ}$ pulse width was $3.7 \mu \mathrm{s}$. A total of 2048 signal transients were accumulated with $6 \mathrm{~s}$ relaxation delays at ambient temperature. All spectra were referenced to external signal as a secondary reference (frequency peak at $38.5 \mathrm{ppm}$ with respect to TMS).

\subsection{Boiling experiment}

Disc shape $(\sim 2 \mathrm{~cm}$ in diameter) autoclaved hydrogels and freeze-thaw hydrogels were immersed in boiling water for over 2 hours; observations were registered using a digital camera.

\subsection{Swelling}

Swelling of the autoclaved hydrogels was measured in deionized water, in an ion-containing solution ( $8.298 \mathrm{~g}$ of sodium chloride and $0.368 \mathrm{~g}$ of calcium chloride dissolved in $1 \mathrm{~L}$ of deionized water) to simulate wound fluid, and in phosphate buffer (PBS) at $37{ }^{\circ} \mathrm{C}$. The hydrogels were kept in excess media for 11 days and weighed regularly to calculate the swelling ratio (SR in\%) using the following formula:

$$
\mathrm{SR}=\left(W_{\mathrm{s}}-W_{\mathrm{i}}\right) / W_{\mathrm{i}}
$$

where $W_{\mathrm{s}}$ is the weight of the swollen gel and $W_{\mathrm{i}}$ is its initial weight.

\subsection{Mechanical properties}

Mechanical properties of the hydrogels were studied with the autoclaved samples. Tensile tests were conducted using a Texture Analyser XT Plus (Stable Micro Systems Ltd, UK) in sample stretching mode. Rectangular hydrogel samples of 20 $\mathrm{mm}$ in length and $3 \mathrm{~mm}$ in width were used with a 'grip-to-grip' separation of $30 \mathrm{~mm}$. Each sample was subjected to an extension at $100 \mathrm{~mm} \mathrm{~min}^{-1}$ to its breaking point.

\subsection{Scanning electron microscopy (SEM)}

The gels were synthesized, freeze-dried and cut before gold sputter coating. Scanning electron microscopy $(1000 \times$ and $10000 \times$ magnification was used) was carried out using an (FEI Quanta FEG 600 Environmental Scanning Electron Microscope ESEM).

\subsection{Adhesion test}

Adhesion tests were carried out using a Texture Analyser XT Plus (Stable Micro Systems Ltd, UK). The hydrogel samples $(20 \mathrm{~mm}$ diameters) were attached to the probe and a piece of shaved 
porcine skin (supplied by Vicars \& Son butchers, Reading) was placed on the platform below. Porcine skin from the back of the animal was used for these tests. The tests were performed with the following settings: pre-speed test $1.0 \mathrm{~mm} \mathrm{~s}^{-1}$; test-speed 0.5 $\mathrm{mm} \mathrm{s}^{-1}$; post-test speed $1.0 \mathrm{~mm} \mathrm{~s}^{-1}$; applied force $0.5 \mathrm{~N}$; contact time $60.0 \mathrm{~s}$; trigger type auto; trigger force $0.1 \mathrm{~N}$; and return distance of $10.0 \mathrm{~mm}$. Force versus distance curves were recorded using the Texture Analyser software (T.A. Exponent). The samples were put in contact with the porcine skin for $1 \mathrm{~min}$ and then detached in order to collect the following data: maximum force required for the detachment (adhesive strength), total work of adhesion (represented by the area under the force versus distance curve) and the cohesiveness (maximum distance travelled by the gel to be detached from the porcine skin) as defined in Boateng et al. ${ }^{14}$

\subsection{Antimicrobial activity}

The disk diffusion method ${ }^{15}$ was employed to evaluate the antimicrobial properties of all PVA-Gantrez ${ }^{\circledR}$ AN hydrogel samples. Staphylococcus aureus NCTC 8532 strain from the National Collection of Type Cultures was used in these experiments. Nutrient agar and nutrient broth were prepared following manufacturer instructions and were sterilized by autoclave $\left(121{ }^{\circ} \mathrm{C}\right.$ for 15 minutes). Staphylococcus aureus was grown overnight $\left(37{ }^{\circ} \mathrm{C}\right.$ with rotary shaking at $\left.200 \mathrm{rpm}\right)$ in nutrient broth. Serial dilutions were performed to determine initial cell numbers and testing concentrations. Nutrient agar plates were inoculated with a Staphylococcus aureus concentration dose of either $10^{8} \mathrm{CFU} \mathrm{mL}{ }^{-1}$ or $10^{4} \mathrm{CFU} \mathrm{mL}{ }^{-1}$ spread onto agar surface. Disks of hydrogel sample $(\sim 1 \mathrm{~cm}$ in diameter $)$ were cut and placed on the agar surface and incubated at $37^{\circ} \mathrm{C}$ for 24 hours. The diameter of the growth inhibition zone was measured using a ruler. Six samples and three repeats each were tested in parallel for each experiment. All experiments were performed aseptically.

\subsection{Cytotoxicity analysis}

The MTT (3-(4,5-dimethylthiazol-2-yl)-2,5-diphenyl tetrazolium bromide) assay was performed to indirectly test the cytotoxicity of the hydrogel samples. ${ }^{16-19}$ The hydrogel samples $(\sim 5 \mathrm{mg}$ each) were immersed in $8 \mathrm{~mL}$ of Dulbecco's modified Eagle's medium (DMEM from Sigma) containing $2 \% \mathrm{v} / \mathrm{v}$ L-glutamine and $50 \mu \mathrm{g} \mathrm{mL}{ }^{-1}$ gentamicin, and incubated at $37^{\circ} \mathrm{C}$. On the $1 \mathrm{st}$, 2nd and 7th day of the experiment, media was removed and collected (these represent the gel extracts) in sterile conditions and replaced with fresh media. A commercial wound dressing (Aquacel® Ag by ConvaTec Ltd) was used as a control ( $\sim 5 \mathrm{mg}$ ).

Human dermal fibroblasts passage 3 were seeded at a density of $8 \times 10^{4}$ cells per $\mathrm{mL}$ in complete medium $(10 \%$ fetal bovine serum (FBS), $2 \%$ L-glutamine and $50 \mu \mathrm{g} \mathrm{mL} \mathrm{m}^{-1}$ of gentamicin were added) in a sterile 96-wells culture plate and cultured until confluence was reached. The medium was then replaced with the hydrogel (and control) extracts and incubated at $37^{\circ} \mathrm{C}$ and $5 \% \mathrm{CO}_{2}$ for $24 \mathrm{~h}$. The MTT solution was prepared in warm PBS $\left(0.5 \mathrm{mg} \mathrm{mL}^{-1}\right)$ and added to the plate $(100 \mu \mathrm{L}$ per well), which was incubated at $37{ }^{\circ} \mathrm{C}$ for $3 \mathrm{~h}$. The MTT and medium was removed and $100 \mu \mathrm{L}$ of DMSO were used to solubilize the formazan retained by the cells. The absorbance was measured with a Biotek Synergy HT detector at 570/630 nm.

Human fibroblasts were also seeded at the same density $(8 \times$ $10^{4}$ cells per $\mathrm{mL}$ ) in complete medium in the presence of $\sim 5 \mathrm{mg}$ hydrogel samples for direct biocompatibility testing. A hydrogel sample prepared by autoclaving PVA-Gantrez $\AA$ AN aqueous mixtures $(1: 1)$ containing $0.05 \% \mathrm{v} / \mathrm{v}$ Triton ${ }^{\circledR}$ was used as a positive control; this sample is expected to cause significant cytotoxicity. A commercial Aquacel® $\mathrm{Ag}$ (ConvaTec Ltd) dressing was used as a negative control. The cells were cultured and the media changed every day. Culture growth was monitored and the cells were stained for nuclei fluorescence using Hoescht 33342 and the Zeiss A1 Inverted Epifluorescent Microscope with Nikon NIS Elements photo-capturing system was used to take images of the cultures.

\subsection{Statistical analysis}

One-way ANOVA with Bonferroni post hoc test on Prism (Graphpad, USA) was used to carry out all the statistical analysis to compare different groups of data.

\section{Results and discussion}

\subsection{Synthesis of hydrogels and mechanism of cross-linking in polymer mixtures}

Transparent, bubble-free, flat hydrogel samples were successfully produced from aqueous mixtures of PVA and Gantrez® AN using autoclaving. In order to establish the nature of crosslinking in the mixtures of Gantrez ${ }^{\circledR}$ AN and PVA, the autoclaved hydrogel samples were freeze-dried and subjected to FTIR (Fig. 1) and solid state ${ }^{13} \mathrm{C}$ CP-MAS NMR (Fig. 2) for spectroscopic characterisation. The results were compared with the spectra of the individual polymers.

In the FTIR spectrum of Gantrez ${ }^{\circledR}$ AN the absorption peak of the anhydride carbonyl group is clearly visible in the region $1760-1820 \mathrm{~cm}^{-1}$; OH stretch peak $\left(3300-2500 \mathrm{~cm}^{-1}\right)$ and CO stretch peak typical for carboxylic acids $\left(1320-1000 \mathrm{~cm}^{-1}\right)$ are visible as well as the $\mathrm{OH}$ bend peak characteristic for carboxylic groups at $950-910 \mathrm{~cm}^{-1}$; C-O-C stretch peak of the methyl vinyl ether unit is visible at $1092 \mathrm{~cm}^{-1} .^{20}$ In the PVA spectrum it is

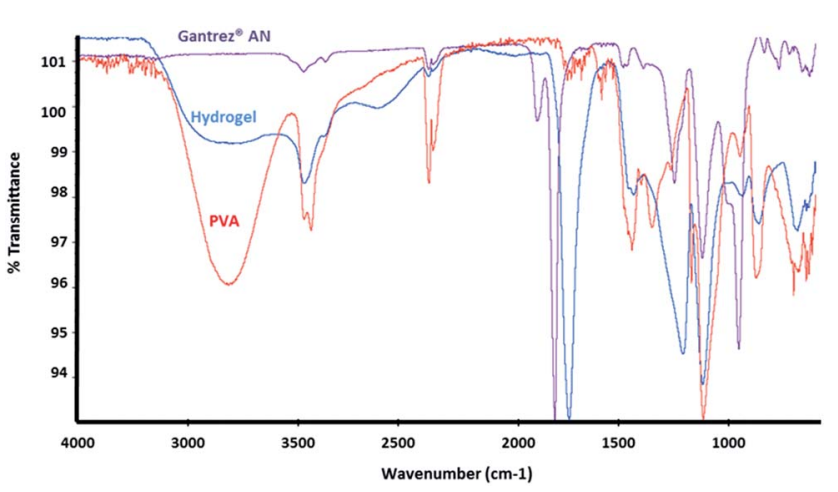

Fig. 1 FTIR spectra of Gant powder (purple), PVAm powder (red) and freeze-dried PVAm-Gant (1:1) hydrogel sample (blue). 


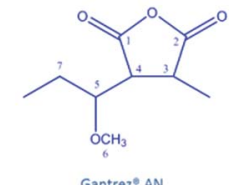

150
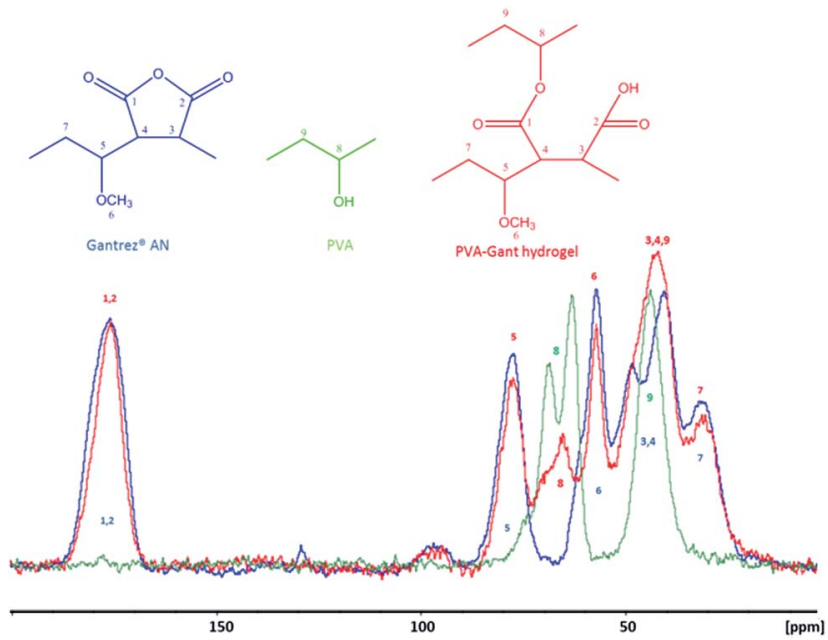

Fig. 2 Solid state ${ }^{13} \mathrm{C}$ CP-MAS NMR spectra of Gant, PVAm and PVAm-Gant $(1: 1)$ hydrogel sample.

possible to observe the characteristic peaks of the hydroxyl groups $\left(3200-3400 \mathrm{~cm}^{-1}\right)$, CH stretch $\left(3000-2850 \mathrm{~cm}^{-1}\right), \mathrm{CH}$ bend (1470-1450 $\mathrm{cm}^{-1}$ ) and CO stretch peak of alcohol groups $\left(1320-1000 \mathrm{~cm}^{-1}\right)$. The peaks present in the freeze-dried hydrogel spectrum result from the spectral features of both polymers. For instance, in the hydrogel's spectrum the peak attributable to the carbonyl of the maleic anhydride is shifted to lower wavelengths, typical for the carbonyl of the carboxylic acid $\left(1710 \mathrm{~cm}^{-1}\right)$ or the ester $\left(1735 \mathrm{~cm}^{-1}\right)$. The peak typical for the hydroxyl groups of the PVA is present in the same region 3200$3400 \mathrm{~cm}^{-1}$ in the hydrogel's spectrum as well, even if less pronounced. The peak at $1172-1177 \mathrm{~cm}^{-1}$ could support the esterification reaction between the $\mathrm{COOH}$ formed after the ring opening and the $\mathrm{OH}$ groups of PVA. ${ }^{20} \mathrm{~A}$ peak at $2400-2300 \mathrm{~cm}^{-1}$ is visible in all the three spectra and it is attributable to carbon dioxide present in the atmosphere. ${ }^{13}$ Fig. 2 shows the solid state ${ }^{13} \mathrm{C}$ CP-MAS NMR spectra of Gantrez ${ }^{\circledR}$ AN, PVA and freeze-dried hydrogel sample with related assignments.

In the spectrum of Gantrez ${ }^{\circledR} \mathrm{AN}: \mathrm{C} 1$ and $\mathrm{C} 2$ of the two carbonyl groups of the anhydride cycle give a single peak at 190170 ppm; C3 and C4 of the maleic anhydride cycle give a double peak at 35-55 ppm; $\mathrm{C} 5$ and $\mathrm{C} 7$ of the lateral chain give signals at 85-70 and 25-35 ppm, respectively; C6 of the methyl group gives a peak at $60-50 \mathrm{ppm}$. In the PVA spectrum: C8 gives a double peak at 70-60 ppm and C9 a single one at 50-40 ppm.

Moving on to the hydrogel's spectrum: $\mathrm{C} 1$ and $\mathrm{C} 2$ of the now open maleic cycle give a single peak in the same region (190-170 ppm) as in the spectrum of Gantrez ${ }^{\circledR} \mathrm{AN}$, as well as C3 and C4 that in this case give a higher peak at $55-35 \mathrm{ppm}$ (for the contribution of C9), C5 (85-70 ppm), C6 (60-50 ppm) and C7 (25-35 ppm). The peak given by $\mathrm{C} 8$ in the hydrogel's spectrum is still found at 70-60 ppm (as in PVA) but is now single.

Since the spectroscopy did not provide a conclusive evidence on the formation of covalent linkages between Gantrez® AN and PVA, additional experiments were conducted with PVAGantrez ${ }^{\circledR}$ AN hydrogels cross-linked via non-covalent interactions through the formation of hydrogen bonds and crystalline domains. These physically cross-linked samples were prepared using freeze-thawing procedure, well-known for the preparation of hydrogels from PVA. ${ }^{21}$ For this purpose solution mixtures of Gantrez ${ }^{\circledR}$ AN and PVA, containing $0.25 \% \mathrm{w} / \mathrm{v}$ of $\mathrm{NaOH}$, were frozen for a period of 9 hours and then thawed at room temperature leading to opaque hydrogels. Immersion of hydrogels produced via the freeze-thaw technique into boiling water results in completely different phenomena to the hydrogels produced via the autoclaving technique. The physically cross-linked freeze-thaw hydrogels undergo quick dissolution within 20 seconds due to disruption of intermolecular hydrogen bonds at high temperatures (Fig. 3). On the other hand, the autoclaved hydrogels are not dissolved upon immersion into boiling water and only minor additional swelling occurred within two hours. The results of the boiling experiments clearly demonstrate that autoclaving leads to the formation of stable bonds between Gantrez ${ }^{\circledR}$ AN and PVA, whose nature is likely to be covalent. We propose that autoclaving leads to the formation of ester bonds between the carboxylic groups of Gantrez ${ }^{\circledR}$ AN and hydroxyl groups of PVA. These bonds are not detected by spectroscopic techniques because their concentration in the hydrogels is very low. This is also consistent with our previous report on the synthesis of hydrogels using microwave processing. ${ }^{13}$ Typically $1-2$ ester bonds per macromolecule will be sufficient to make a network and the concentration of these new bonds will be very low and impossible to detect by spectroscopic techniques. The presence of these bonds is further confirmed by degradation and complete dissolution of autoclaved hydrogels in boiling $10 \% \mathrm{NaOH}$ solution within approximately 45 minutes.

It should also be noted that autoclaving solutions of individual PVA and Gantrez ${ }^{\circledR}$ AN does not result in formation of hydrogels. Autoclaving-mediated cross-linking is only possible when two polymers are present in an aqueous mixture, which further confirms the formation of ester bonds between carboxylic groups of Gantrez® AN and hydroxyl groups of PVA.

Depending on the nature of cross-linking, the same mixture of polymers can be used to produce hydrogels with completely different properties (Fig. 4). For instance, physically crosslinked hydrogels produced by freeze-thaw technique are not fully transparent, have reversible properties and form at low temperatures, making them promising for tissue engineering
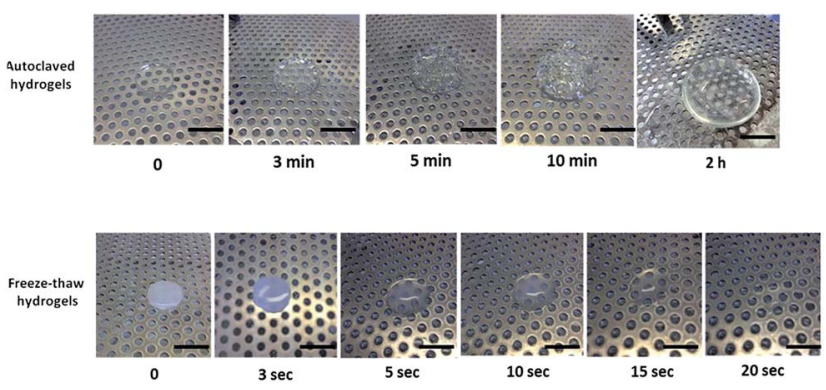

Fig. 3 Comparison between the behaviors of chemically and physically cross-linked PVAm-Gant (1:1) hydrogels after being immersed in boiling water. Scale bars are $2 \mathrm{~cm}$. 


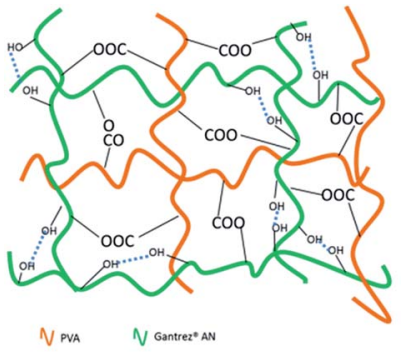

(a)

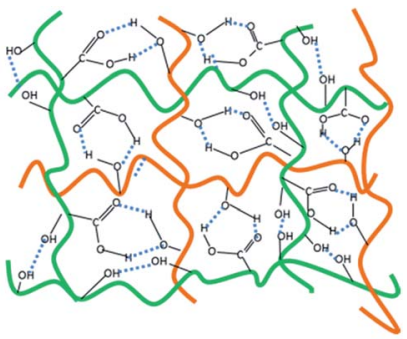

(b)
Fig. 4 Proposed structure of chemically cross-linked autoclaved hydrogels (a) and physically cross-linked freeze-thaw hydrogels (b) based on PVA-Gant mixtures.

and drug delivery due to their excellent mechanical strength. ${ }^{22}$ Chemically cross-linked autoclaved hydrogels, on the contrary, are less suitable for tissue engineering applications because of high temperatures used for their preparation; but they are highly promising for wound care. The properties of these hydrogels and their applicability for wound care shall be discussed in detail.

\subsection{Physicochemical properties of autoclaved hydrogels}

The autoclaved hydrogels immersed in deionized water undergo significant swelling, taking over $100 \mathrm{~g}$ of water per $1 \mathrm{~g}$ of initial sample within 11 days (Fig. 5). Even after this relatively long period of time the hydrogels have not yet reached their maximum swelling capacity and continue to absorb water. The swelling ratio (SR) of the autoclaved hydrogels was significantly higher in deionized water compared to the ion-containing solution which was used in order to more closely resemble the ionic strength of wound fluid. Only a modest 5-times swelling was observed in the ion-containing solution. This significant reduction in SR with the ion-containing solution

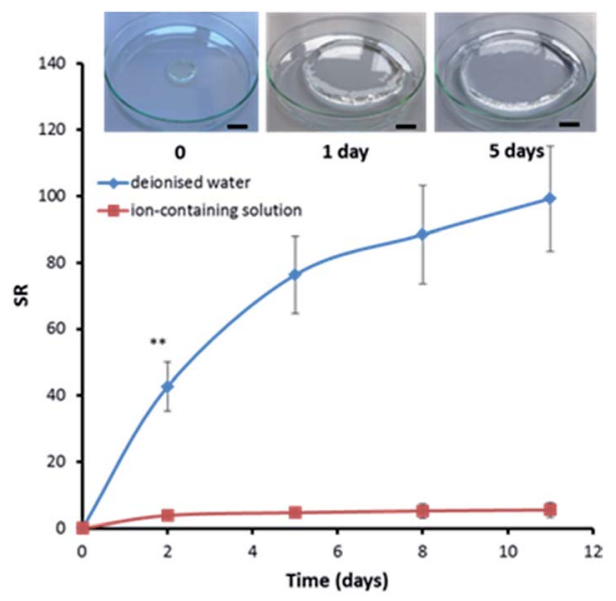

Fig. 5 Swelling kinetics for PVAm-Gant (1:2) in deionized water and in an ion-containing solution at room temperature. Insert: photograph of a hydrogel undergoing swelling in deionized water. The scale bar shown is $2 \mathrm{~cm}$. Swelling curves for other hydrogel compositions could be found in Fig. S1 and S2. $\dagger$ compared to deionized water is typical for polyelectrolyte hydrogels and can be explained by the charge screening effects and the lower osmotic pressure due to the difference in mobile ions present between the hydrogel matrix and the external medium. ${ }^{23}$

No statistically significant effects of the polymer ratios of PVA molecular weights on the swelling ratios were observed $(p<$ $0.05)$. It should be noted that all the values have high standard deviations because of the high amount of surface water present which was impossible to fully remove during the gravimetric measurements.

The mechanical behaviour of the autoclaved hydrogels was evaluated using a tensile test (Table 1 ). The exemplary strengthstrain curves are shown in Fig. S3. $\dagger$ Young's modulus is commonly defined as the slope of stress (the force applied) versus strain (the deformation occurred in the material) curve. It is considered as the most relevant mechanical property of biomaterials. ${ }^{24}$ The ultimate strength is the maximum stress that a material can withstand. The elongation to break is the deformation (strain) measured at fracture..$^{25}$

The autoclaved hydrogels exhibited suitable mechanical properties for the proposed application. Similar materials designed for wound dressing or other biomedical applications, already described in the literature, exhibit higher stiffness compared with these hydrogels. ${ }^{26,27}$ For instance, the Young's modulus of the bacterial cellulose (nf-BC)-PVA composite hydrogels proposed by Qiao et al. ${ }^{26}$ are between 0.5 and $1 \mathrm{MPa}$. Hydrogels have often been compared to living biological tissue for their elasticity and flexibility and are considered for this reason as a very good temporary scaffold for tissue repair. ${ }^{28,29} \mathrm{All}$ samples tested, showed more desirable values of elongation to break than the normal human skin itself, which goes from $75 \%$ at birth to $60 \%$ in the elderly. ${ }^{30}$ Young's modulus and tensile stress at break data are similar for all samples tested (Table 1). 1 to 1 molar ratio compositions exhibited higher strength values, with the gel containing high $M_{\mathrm{w}}$ PVA being the most elastic among the formulations considered (with an elongation to break of $159 \pm 6 \%$ ).

The porous structure of freeze-dried hydrogels was studied using SEM (Fig. 6). We observed highly porous structures with different pore sizes, wall thickness and distribution. Both large and small pores are clearly visible in these samples, indicating their inhomogeneous nature.

\subsection{Adhesion to skin}

Commercial wound dressings are often classified as adherent, low-adherent and non-adherent materials. ${ }^{31}$ In fact, the level of adhesion represents an important characteristic of the product and it may significantly affect the healing process. ${ }^{32}$ Depending on the type of the wound and stage of the healing a different degree of adherence could be required. The newly formed tissue and peri-wound skin is fragile and an extremely adherent dressing could cause further trauma and pain upon its removal. $^{31,33,34}$

At the same time, it is convenient to have a dressing capable of remaining in place for a number of days in order to protect 
Table 1 Mechanical properties of hydrogel samples. Data are shown as mean \pm standard deviation. Statistical significance $(p<0.05)$ was calculated for the data related to Young's modulus, tensile stress at break, ultimate strength and elongation to break of the hydrogel compositions $(a-f)$. Letters after each value indicate which other composition the sample is significantly different from

\begin{tabular}{lllll}
\hline $\begin{array}{l}\text { Composition } \\
\left(\mathrm{mol} \mathrm{mol}^{-1}\right)\end{array}$ & $\begin{array}{l}\text { Young's modulus } \\
\left(\mathrm{N} \mathrm{mm}^{-2}\right)\end{array}$ & $\begin{array}{l}\text { Tensile stress at break } \\
\left(\mathrm{N} \mathrm{mm}^{-2}\right)\end{array}$ & $\begin{array}{l}\text { Ultimate strength } \\
\left(\mathrm{N} \mathrm{mm}^{-1}\right)\end{array}$ & $\begin{array}{l}\text { Elongation to break } \\
(\%)\end{array}$ \\
\hline${ }^{\mathrm{a}}$ PVAm-Gant $(1: 1)$ & $(4.6 \pm 1.2) \times 10^{-5}$ & $(3.1 \pm 0.3) \times 10^{-3}$ & $(6.4 \pm 0.8) \times 10^{-2 \mathrm{e}}$ & $110 \pm 17$ \\
${ }^{\mathrm{b}}$ PVAm-Gant $(1: 2)$ & $(1.3 \pm 1.5) \times 10^{-4}$ & $(2.6 \pm 0.2) \times 10^{-3}$ & $(5.1 \pm 0.5) \times 10^{-2 \mathrm{~d}}$ & $79 \pm 13^{\mathrm{d}}$ \\
${ }^{\mathrm{c}}$ PVAm-Gant $(2: 1)$ & $(1.2 \pm 1.5) \times 10^{-4}$ & $(9.7 \pm 12.0) \times 10^{-3}$ & $(4.9 \pm 1.3) \times 10^{-2 \mathrm{~d}}$ & $96 \pm 32$ \\
${ }^{\mathrm{d}}$ PVAh-Gant $(1: 1)$ & $(3.0 \pm 2.0) \times 10^{-5}$ & $(4.7 \pm 0.1) \times 10^{-3}$ & $(9.3 \pm 2.0) \times 10^{-2 \mathrm{e}}$ & $159 \pm 6^{\mathrm{e}}$ \\
${ }^{\mathrm{e}}$ PVAh-Gant $(1: 2)$ & $(2.3 \pm 1.1) \times 10^{-5}$ & $(1.4 \pm 1.0) \times 10^{-3}$ & $(2.8 \pm 2.0) \times 10^{-2 \mathrm{f}}$ & $87 \pm 44$ \\
${ }^{\mathrm{f}}$ PVAh-Gant $(2: 1)$ & $(4.3 \pm 0.5) \times 10^{-5}$ & $(3.3 \pm 0.2) \times 10^{-3}$ & $(6.8 \pm 0.4) \times 10^{-2}$ & $101 \pm 2$
\end{tabular}

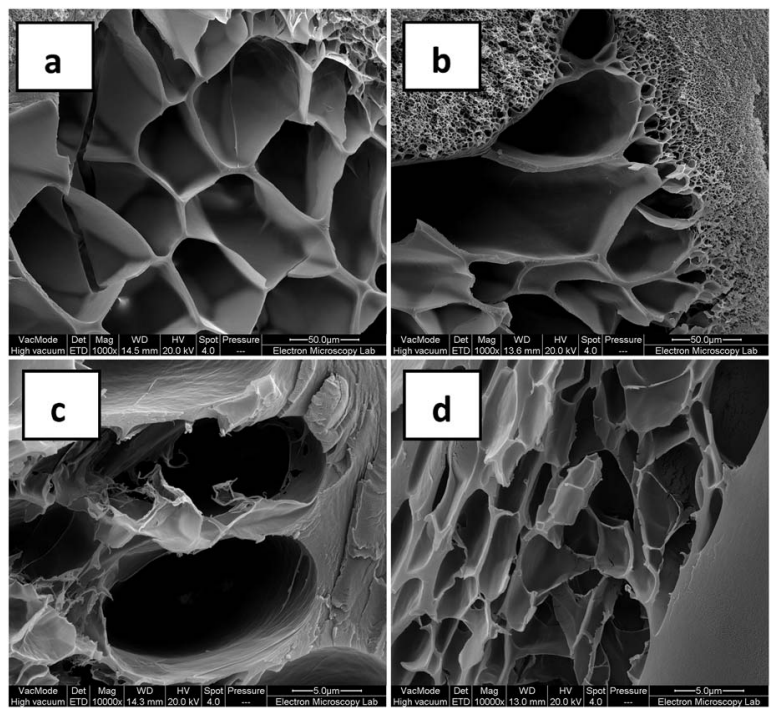

Fig. 6 SEM images of freeze-dried hydrogels: (a and c) PVAh-Gant (1: 1); (b and d) PVAh-Gant (1: 2). Scale bar is $50 \mu \mathrm{m}$ ( $a$ and $b)$ and $5 \mu \mathrm{m}$ (c and d).

the wound from bacteria and other pathogens. Porcine skin was used as a model to test the adhesion of the autoclaved hydrogels. The study of hydrogel detachment from skin surface can provide several characteristics of adhesion, including the force of detachment, the work of adhesion and cohesiveness. ${ }^{14}$

Fig. 7 shows the summary of detachment characteristics for the hydrogels prepared with different polymer ratios and from different PVA samples. The results of the detachment force, work of adhesion and adhesiveness are in agreement with each other and indicate that the hydrogels prepared with different polymer ratios have difference in their adhesive characteristics.

The samples prepared from lower molecular weight PVA $(51.3 \mathrm{kDa})$ show greater adhesion when they contain an excess of PVA. On the contrary, the hydrogels composed of higher molecular weight PVA (66.3 kDa) showed the greatest adhesion when the $1: 1$ polymer ratio was used.

Adhesion is a complex phenomenon that is still not completely understood. It has been suggested that adhesion is directly related to the formation of non-covalent bonds, such as hydrogen bonds, between two surfaces in contact. ${ }^{35}$ Additionally, it is believed that the diffusion of unbound or loosely bound macromolecules, such as free linear polymer present, into the outer layer of the epithelium could also contribute to the adhesion. Therefore by increasing the degree of crosslinking which results in fewer loose macromolecules with potential to diffuse should decrease the adhesion to skin. Skin condition is expected to substantially affect adhesion of hydrogels. In this work, a healthy intact porcine skin was used as a substrate to study adhesive properties of hydrogels. The measurements of hydrogel adhesion using intact skin provide better reproducibility of results; therefore, this model is more common in characterisation of wound care products. If the skin was damaged, completely different adhesion results are expected as the wound surface will have substantially different nature and the results are expected to be less reproducible.

\subsection{Antimicrobial properties}

It is very important to protect the wound from bacteria and pathogens that could cause further damage and delay the healing process; especially when the injury is chronic, large in size or the patient is particularly at risk, for example, in severe burns. ${ }^{36,37}$ For this reason, many products containing antimicrobial agents are already available on the market, such as Aquacel Ag® (by ConvaTec Ltd.) and Acticoat ${ }^{\mathrm{TM}}$ (by Smith \& Nephew). ${ }^{38,39}$ Ionic silver $\left(\mathrm{Ag}^{+}\right)$has become very popular as an antimicrobial since the late 1960's when it was often used in association with sulfadiazine (silver sulfadiazine SSD). Several mechanisms were proposed for its antimicrobial action, such as the disruption of hydrogen bonding in bacterial DNA resulting in denaturation and its well-known ability to bind thiol groups $(-\mathrm{SH})$ present on the surfaces of biological molecules such as enzymes, causing their inactivation. ${ }^{40-42}$ Another antimicrobial agent frequently used in wound management is molecular iodine $\left(\mathrm{I}_{2}\right)$, which damages the cell walls of microorganism leading to leakage of the cell contents and cessation of protein synthesis. Both effects result in cell death. ${ }^{43,44}$

The antimicrobial properties of the PVAm-Gant and PVAhGant hydrogels were tested in a $24 \mathrm{~h}$ growth inhibition assay against a suitable Gram positive strain, Staphylococcus aureus. This pathogen is widely present in the environment and is responsible for many skin infections. ${ }^{45}$ Commercial wound dressing Aquacel® $\mathrm{Ag}$ was evaluated in parallel as a control. This method allows an easy and clear evaluation of antimicrobial activities of drugs or biomaterials and it is commonly used 
(b)

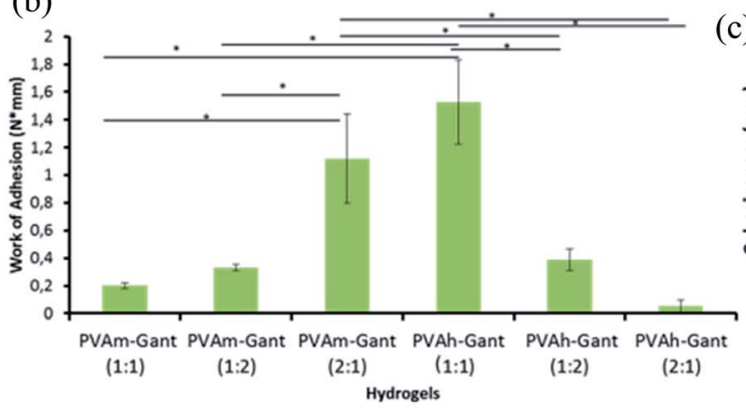

(c)

(a)

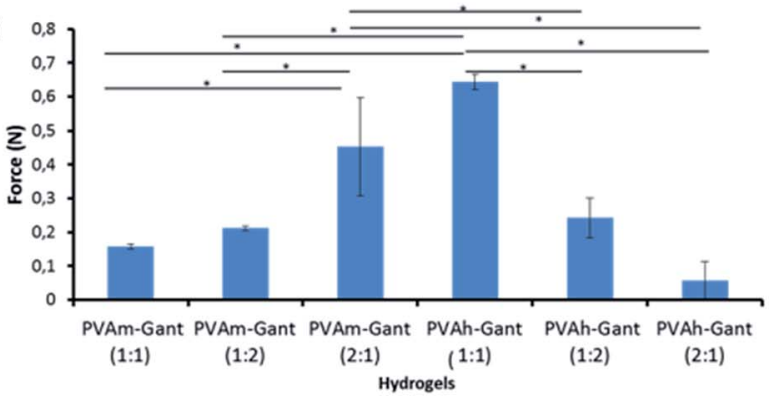

(c) 9

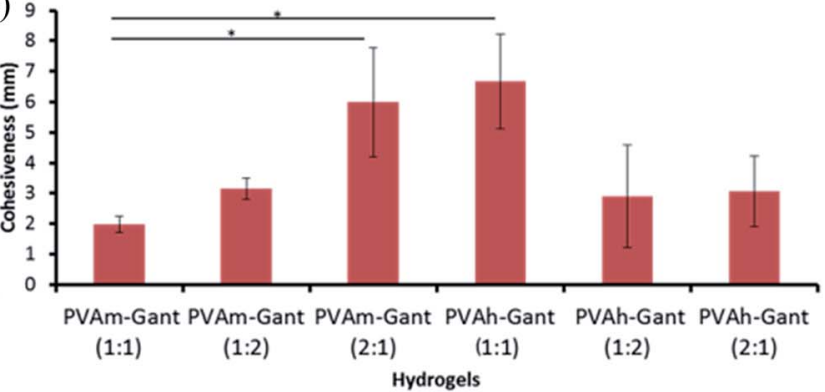

Fig. 7 Adhesion of hydrogels to porcine skin: force of detachment values (a), work of adhesion value (b) and cohesiveness values (c) for autoclaved hydrogels. Asterisk $(*)$ indicates significant statistical difference $(p<0.05)$. Typical detachment profile can be seen in Fig. S4. $\dagger$

in microbiology, as it gives a correlation between concentration and bacterial inhibition. ${ }^{\mathbf{1 4 , 4 6}}$

A clear bacteria growth inhibition zone (Fig. 8) was observed for all gel samples, confirming their intrinsic antimicrobial properties. As expected, it was higher when the bacteria concentration was lower $\left(10^{4} \mathrm{CFU} \mathrm{mL}{ }^{-1}\right)$. All hydrogel samples showed Staphylococcus aureus growth inhibition comparable to

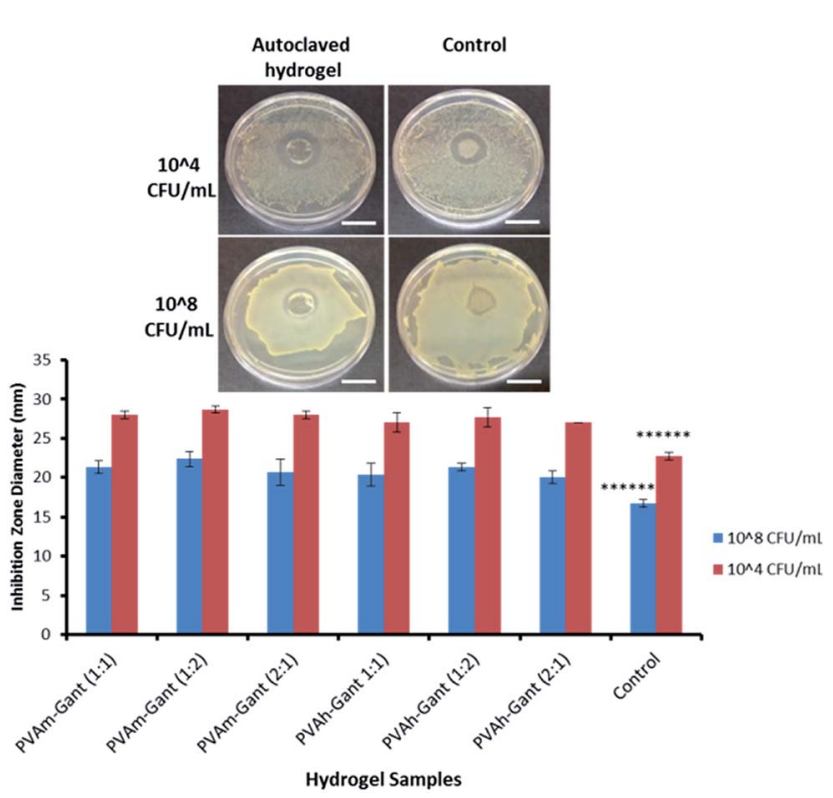

Fig. 8 Antimicrobial activity of hydrogel samples (left) and control (right) against $\mathrm{S}$. aureus $\left(10^{4}\right.$ and $\left.10^{8} \mathrm{CFU} \mathrm{mL}{ }^{-1}\right)$. The images on the top were taken during the antimicrobial test. Aquacel ${ }^{\circledR} \mathrm{Ag}$ (ConvaTec Ltd.) was used as control. Clear zones of growth inhibition were visible around the samples and the control. Scale bar is $2 \mathrm{~cm}$. Asterisk (*) indicates significant statistical difference $(p<0.05)$. the control. The antimicrobial activity of hydrogels containing PVA has not been reported previously. However, Gantrez@ AN has some documented antimicrobial properties. ${ }^{47}$ To establish the nature of the antimicrobial activity in autoclaved hydrogels we have prepared micronized dispersions of these samples and measured their $\mathrm{pH}$. It was found that all samples exhibit $\mathrm{pH}$ of $3-4$. This acidity may result from carboxylic groups formed by opening of maleic anhydride cycles and also from traces of acetic acid ( $>0.01 \%$ as estimated using ${ }^{1} \mathrm{H}$ NMR spectroscopy) present in autoclaved samples. Acidic pH is known to inhibit the growth of Staphylococcus aureus, which is likely to explain the antimicrobial properties of these hydrogels. ${ }^{48}$

\subsection{Cytotoxicity analysis}

An MTT (3-(4,5-dimethylthiazol-2-yl)-2,5-diphenyl tetrazolium bromide) assay was performed to determine the indirect

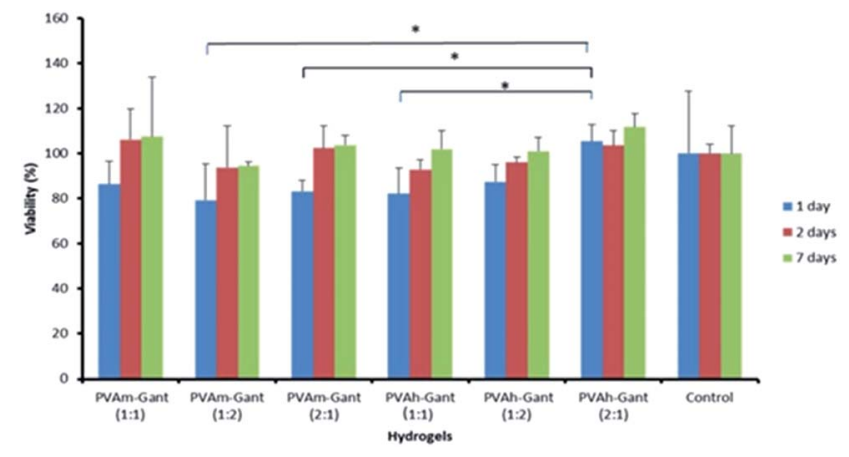

Fig. 9 Indirect cytotoxicity assessment of hydrogels. Cell seeding density was $8 \times 10^{4}$ cells per $\mathrm{mL}$. Cell viability from MTT test performed using the hydrogels produced by autoclaving. Asterisk (*) indicates a significant difference $(p<0.05)$. 
1 day
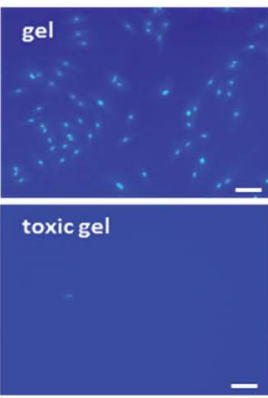
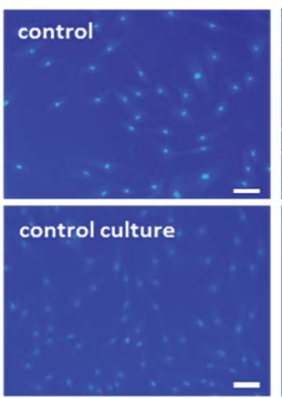

2 days
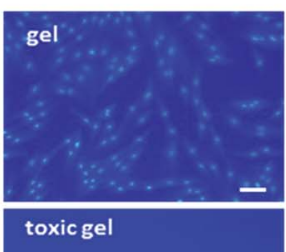
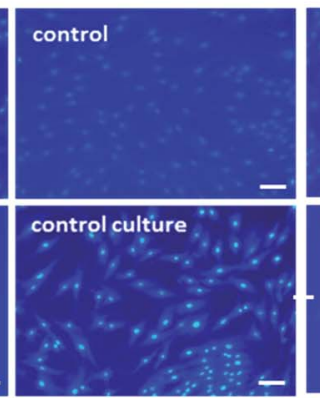

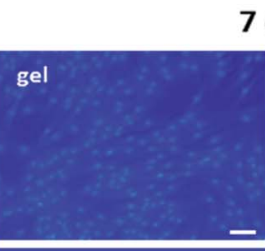

toxic gel
7 days

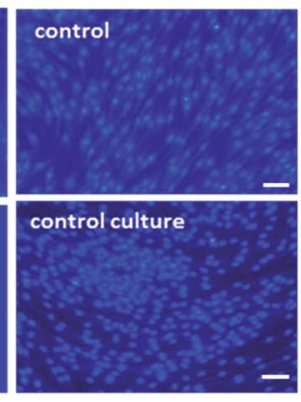

Fig. 10 Images of cell cultures stained for nuclei fluorescence after 1, 2 and 7 days. The cells were seeded in the presence of autoclaved PVAmGant $(1: 1)$ hydrogel samples, a commercial product and a gel containing cytotoxic $0.05 \%$ of Triton®. Scale bars are $100 \mu \mathrm{m}$. A control culture was monitored as well.

cytotoxicity of PVA-Gantrez® AN hydrogel samples. This colorimetric, quantitative method provides a rapid evaluation of cell viability and has now been considered as a standard technique to evaluate toxicological properties of various materials. ${ }^{15}$

The tetrazolium salt is positively charged and can penetrate viable eukaryotic cells where it is converted into a colored product (formazan) with a maximum absorbance near 570 $\mathrm{nm} .{ }^{49}$ The test was carried out using extracts collected from the gels in sterile conditions (Fig. 9). The MTT assay indicated that the extracts collected at 24 and $48 \mathrm{~h}$ from the hydrogels gave $>70 \%$ cell viability. These levels of cell viability indicate that the hydrogels could be considered biocompatible for the application as medical devices. ${ }^{50}$

To assess the direct cytotoxicity human fibroblasts were seeded in the presence of the autoclaved hydrogels (Fig. 10). The gel produced in the presence of $0.05 \%$ Triton $\circledast$ served as a positive control and killed all the cells as expected. The cultures were stained for nuclei fluorescence and images were taken at 1, 2 and 7 days post-seeding. Cells growth and proliferation were comparable in the presence of the samples and the commercial product used as a control. Fibroblasts found favorable conditions to proliferate and did not show a modified behavior when seeded in the presence of PVA-Gantrez® AN autoclaved hydrogels.

\section{Conclusions}

Autoclaving aqueous mixtures of PVA and Gantrez ${ }^{\circledR}$ AN is a novel method for the production of hydrogels, resulting in sterile, antimicrobial and biocompatible materials with very good swelling capability, excellent mechanical and adhesive properties. These hydrogels do not require any post purification because no monomers, toxic cross-linkers or initiators were used in their synthesis. This method allows the rapid, straightforward synthesis of hydrogel samples having different shapes and volumes, depending on the final application required and on the capacity of the autoclave used. Costeffective and relatively safe production steps in comparison with alternative cross-linking techniques provide significant advantages for the industrial production of these materials.
The materials produced are proposed for the development of novel wound dressings. The antimicrobial properties of the autoclaved hydrogels, which therefore do not require the addition of any antimicrobial agents such as silver or iodine, represent a great advantage for application in wound management.

The full transparency of the hydrogels is an additional advantage allowing visual monitoring of the wound without removing the dressing so that it can be left in place for a longer period of time, reducing the risk of contamination to the wound from the environment.

\section{Acknowledgements}

The authors acknowledge the University of Reading and ConvaTec Ltd for funding this research project. Chemical Analysis Facility (University of Reading) is acknowledged for access to FTIR, NMR and SEM. Dr Radoslaw M. Kowalczyk is acknowledged for recording solid state NMR spectra and Miss Amanpreet Kaur for her help with SEM. Brett Symonds is thanked for analysis of the molecular weight of PVA samples using GPC and help during the preparation of this manuscript.

\section{References}

1 T. R. Hoare and D. S. Kohane, Polymer, 2008, 49, 1993-2007.

2 C. Elvira, J. Mano, J. San Román and R. Reis, Biomaterials, 2002, 23, 1955-1966.

3 E. Caló and V. V. Khutoryanskiy, Eur. Polym. J., 2014, 65, 252267.

4 N. A. Peppas, B. V. Slaughter and M. A. Kanzelberger, Polymer Science: A Comprehensive Reference, Elsevier, 2012.

5 M. R. Aguilar and J. San Román, Smart Polymers and their Applications, Elsevier, 2014.

6 A. Jones and D. Vaughan, J. Orthop. Nurs., 2005, 8, S1-S11.

7 F. Reyes-Ortega, A. Cifuentes, G. Rodríguez, M. R. Aguilar, Á. González-Gómez, R. Solis, N. García-Honduvilla, J. García-Sanmartin, A. Martínez and J. San Román, Acta Biomater., 2015, 23, 103-115.

8 P. S. Murphy and G. R. D. Evans, Plast. Surg. Int. J., 2012, 2012, 1-7. 
9 V. Jones, J. E. Grey and K. G. Harding, BMJ, 2006, 332, 777780.

10 D. Hofman, Wound Essentials, 2007, 2, 68-73.

11 B. Balakrishnan, M. Mohanty, P. R. Umashankar and A. Jayakrishnan, Biomaterials, 2005, 26, 6335-6342.

12 J. M. Rosiak and F. Yoshii, Nucl. Instrum. Methods Phys. Res., Sect. B, 1999, 151, 56-64.

13 J. P. Cook, G. W. Goodall, O. V. Khutoryanskaya and V. V. Khutoryanskiy, Macromol. Rapid Commun., 2012, 33, 332-336.

14 J. S. Boateng, H. V. Pawar and J. Tetteh, Int. J. Pharm., 2013, 441, 181-191.

$15 \mathrm{H}$. O. Hallander and L. Gunnar, Antimicrob. Agents Chemother., 1972, 422-426.

16 T. Mosmann, J. Immunol. Methods, 1983, 65, 55-63.

17 M. V. Risbud and R. R. Bhonde, Drug Delivery, 2000, 7, 69-75.

18 D. Wang, Biomaterials, 2003, 24, 3969-3980.

19 G. Ciapetti, E. Cenni, L. Pratelli and A. Pizzoferrato, Biomaterials, 1993, 14, 359-364.

20 O. V. Khutoryanskaya, V. V. Khutoryanskiy and R. A. Pethrick, Macromol. Chem. Phys., 2005, 206, 1497-1510. 21 S. R. Stauffer and N. A. Peppas, Polymer, 1992, 33, 3932-3936. 22 V. I. Lozinsky, A. L. Zubov and E. F. Titova, Enzyme Microb. Technol., 1997, 20, 182-190.

23 M. Sadeghi, E. Mohammadinasab and F. Shafiei, Curr. World Environ., 2015, 7, 69-77.

$24 \mathrm{~J}$. W. Nicholson, The Chemistry of Polymers, Royal Society of Chemistry, 2012.

25 ASM International Materials Properties Database Committee, ASM Ready Reference: Properties and Units for Engineering Alloys, ASM International, 1997, ISBN: 0871705850 .

26 K. Qiao, Y. Zheng, S. Guo, J. Tan, X. Chen, J. Li, D. Xu and J. Wuang, Compos. Sci. Technol., 2015, 118, 47-54.

27 M. Ahearne, Y. Yang, A. J. El Haj, K. Y. Then and K.-K. Liu, J. R. Soc., Interface, 2005, 2, 455-463.

28 M. V. Flores-Merino, S. Chirasatitsin, C. Lopresti, G. C. Reilly, G. Battaglia and A. J. Engler, Soft Matter, 2010, 6(18), 4466-4470.

29 S. Dhingra, R. D. Weisel and R.-K. Li, Methods Mol. Biol., 2014, 1181, 51-59.

30 C. Edwards and R. Marks, Clin. Dermatol., 1995, 13, 375-380.

31 M. Rippon, R. White and P. Davies, Wounds UK, 2007, 3, 7686.

32 J. Hurler and N. Skalko-Basnet, J. Funct. Biomater., 2012, 3, 37-48.
33 T. Abdelrahman and H. Newton, Surgery, 2011, 29, 491-495. 34 J. S. Boateng, K. H. Matthews, H. N. E. Stevens and G. M. Eccleston, J. Pharm. Sci., 2008, 97, 2892-2923.

35 Mucoadhesive Materials and Drug Delivery Systems, ed. V. V. Khutoryanskiy, Wiley, 2014.

36 H. Braunwarth and F. H. H. Brill, Wound Medicine, 2014, 5, 16-20.

37 G. Chaby, P. Senet, M. Vaneau, P. Martel, J.-C. Guillaume, S. Meaume, L. Téot, C. Debure, A. Dompmartin, H. Bachelet, H. Carsin, V. Matz, J. L. Richard, J. M. Rochet, N. Sales-Aussias, A. Zagnoli, C. Denis, B. Guillot and O. Chosidow, Arch. Dermatol., 2007, 143, 1297-1304.

38 B. Boonkaew, M. Kempf, R. Kimble, P. Supaphol and L. Cuttle, Burns, 2014, 40, 89-96.

39 J. J. Castellano, S. M. Shafii, F. Ko, G. Donate, T. E. Wright, R. J. Mannari, W. G. Payne, D. J. Smith and M. C. Robson, Int. Wound J., 2007, 4, 114-122.

40 D. J. Leaper, Int. Wound J., 2006, 3, 282-294.

41 M. N. Storm-Versloot, C. G. Vos, D. T. Ubbink and H. Vermeulen, Cochrane Database Syst. Rev. CD006478, 2010, pp. 1-72.

42 D. E. Marx and D. J. Barillo, Burns, 2014, 40(1), S9-S18.

43 H. Vermeulen, S. J. Westerbos and D. T. Ubbink, J. Hosp. Infect., 2010, 76, 191-199.

44 P. M. Mertz, D. A. Marshall and M. A. Kuglar, Arch. Dermatol., 1986, 122, 1133-1138.

45 M. N. Filimon, A. B. Borozan, S. L. Gotia, R. Popescu and V. D. Gherman, An. U.O. Fasc. Biol., 2009, 2, 70-73.

46 S. Marchesan, Y. Qu, L. J. Waddington, C. D. Easton, V. Glattauer, T. J. Lithgow, K. M. McLean, J. S. Forsythe and P. G. Hartley, Biomaterials, 2013, 34, 3678-3687.

47 I. Corzani, US Pat., 6,403,113 B1, January 11, 2002.

48 T. Foster, Staphylococcus, in Med. Microbiol., ed. S. Baron, University of Texas Medical Branch at Galveston, Galveston, USA, 4th edn, 1996, ch. 12.

49 T. L. Riss, R. A. Moravec, A. L. Niles, H. A. Benink, T. J. Worzella and L. Minor, in Cell Viability Assays, 2013, ed. G. S. Sittampalam, N. P. Coussens and H. Nelson, et al., Assay Guidance Manual, Internet, Bethesda, 2004, http://www.ncbi.nlm.nih.gov/books/NBK144065/, accessed February 19, 2015.

50 ISO 10993-5:2009-Biological evaluation of medical devices Part 5, Tests for in vitro cytotoxicity, http://www.iso.org/iso/ catalogue_detail.htm?csnumber $=36406$, accessed February 19, 2015. 